\title{
Variations
}

Variations

Revue internationale de théorie critique

$20 \mid 2017$

Expériences oppositionnelles

\section{L'« activisme institutionnel » de Podemos : entre contestation et transformation organisationnelle}

\section{Mathieu Petithomme}

\section{(2) OpenEdition}

\section{Journals}

Édition électronique

URL : http://journals.openedition.org/variations/833

DOI : 10.4000/variations.833

ISSN : 1968-3960

Éditeur

Les amis de Variations

\section{Référence électronique}

Mathieu Petithomme, «L'« activisme institutionnel » de Podemos : entre contestation et

transformation organisationnelle », Variations [En ligne], 20 | 2017, mis en ligne le 25 avril 2017,

consulté le 25 février 2021. URL : http://journals.openedition.org/variations/833 ; DOI : https://doi.org/

10.4000 /variations.833

Ce document a été généré automatiquement le 25 février 2021.

Les ami•e•s de Variations 


\title{
L'« activisme institutionnel » de Podemos : entre contestation et transformation organisationnelle
}

\author{
Mathieu Petithomme
}

1 Móstoles, 11 mai 2015, meeting de Podemos, campagne des municipales. En cette fin d'après-midi ensoleillée, les orateurs se succèdent à la tribune. Des professeurs, des retraités, des étudiants, des gens d'horizons divers se sont regroupés en plein air sur la dalle du " rastrillo ", le marché ouvert hebdomadaire de la principale ville de la banlieue ouvrière de Madrid. Au-delà du groupe local des militants de Podemos, l'assemblée réunit beaucoup de curieux. Jesús, un chômeur qui «ne vote plus depuis des années », est là "pour voir ce qu'ils disent»: «je veux voir le gourou!», plaisante-t-il, en référence à Pablo Iglesias, qualifié ainsi par certains de ses détracteurs. Beatriz, une infirmière qui s'est jointe au « cercle » de cette profession au sein du parti, l'un des plus actifs, achète un tee-shirt pour sa fille : « je trouve ça bien que des gens normaux se présentent aux élections. Il ne feront peut être pas mieux que les autres, mais ils ne peuvent pas faire pire, vu le nombre de corrompus qu'il y a dans ce pays ! $»^{1}$. L'idée que Podemos serait « le parti des gens ", qu'il permettrait à des citoyens ordinaires de faire de la politique est un argument répété par ses dirigeants, alors que ses adversaires cherchent à le disqualifier en le présentant comme « populiste ».

Mais quelles sont les trajectoires militantes du groupe dirigeant? Sont-ils réellement des citoyens comme les autres et des "profanes $»^{2}$ en politique? Dans quelle mesure Podemos peut-il être considéré comme un parti «populiste " ? Comment parvient-il à articuler les tensions liées à la forme d'un mouvement social, aux impératifs de la compétition électorale et aux nécessités de son institutionnalisation en tant que parti politique? Cet article cherchera ainsi d'abord à préciser les origines de Podemos en tant qu'espace à la confluence des mouvements sociaux et de carrières militantes. Il montrera ensuite que le populisme, loin d'être une caractéristique intrinséque de la nature du parti, constitua il est vrai un outil communicationnelle et discursif initial pour dichotomiser l'espace politique, faire évoluer les lignes de clivage et faciliter son 
émergence. Podemos gouverne aujourd'hui dans de nombreuses mairies, prend des décisions locales et participe au jeu parlementaire national, de telle sorte que même s'il est possible de parler d'une stratégie populiste en campagne, il utilise aussi les ressorts classiques de l'activité politique des partis de gouvernement. Enfin, sa promesse de favoriser l'essor de nouvelles pratiques, par la démocratie d'assemblée dans ses cercles locaux, les primaires ouvertes, une plus grande transparence et des modes de décision plus horizontaux, se heurte aujourd'hui aux nécessités de l'institutionnalisation partisane, ce qui génère des tensions internes. Même si l'on note un renforcement de la centralisation du pouvoir autour du groupe dirigeant, Podemos n'en demeure pas moins un cas par excellence pour étudier les manières avec lesquelles des entrepreneurs politiques peuvent tenter d'instituer une organisation durable, légitimée par des soutiens militants et électoraux, dont les acteurs cherchent à jouer le rôle d'« activistes institutionnels » relayant les revendications des mouvements sociaux au sein même des institutions.

\section{Un parti à la confluence des mouvements sociaux}

3 Si l'on analyse d'abord les trajectoires des cadres de Podemos, on observe qu'il existe une pluralité d'espaces de socialisation politique au sein desquels ses membres les plus influents sont passés, se sont connus, ont organisé des actions collectives et ont acquis des ressources militantes facilitant leur engagement politique postérieur. L'institutionnalisation rapide du parti s'explique d'abord parce que son émergence traduit la confluence d'un ensemble de personnes déjà engagées préalablement dans des carrières militantes, qui se sont impliquées dans différents mouvements sociaux et se sont peu à peu professionnalisées. Pablo Iglesias reconnaît lui-même que " parallèlement au groupe de professeurs et chercheurs de l'université Complutense de Madrid, une nouvelle génération de militants issus de Juventud Sin Futuro (JSF), du mouvement étudiant, de La Tuerka, de secteurs culturels alternatifs et de l'environnement $\mathrm{du} 15-\mathrm{M}$, conformèrent le noyau initial de Podemos $»^{3}$. Hormis quelques exceptions, ses cadres et ses dirigeants n'ont jamais exercé de mandats représentatifs, de telle sorte que l'idée qu'il ne s'agit pas d'un parti de "professionnels» de la politique est vraie. On constate toutefois le rôle central d'« entrepreneurs de cause » impliqués depuis les années 2000 dans les mouvements sociaux, même si cela n'avait pas débouché sur des engagements politiques. Plusieurs facteurs et espaces clés de socialisation méritent d'être évoqués: les expériences partagées d'une "génération précaire ", la politisation au sein de la faculté de science politique de l'université Complutense de Madrid (UCM), les mouvements sociaux antiaustérité et le petit parti d'extrême-gauche Izquierda Anticapitalista.

\section{Du « précariat » à l'engagement militant}

Une première tendance est l'expérience de formes de précarité, qui a constitué un élément déclencheur de l'engagement et d'un type de " politisation incrémentale ${ }^{4}$. Le profil de l'eurodéputée Lola Sánchez (38 ans) est ici assez emblématique. Politologue de formation, elle a immigré en Islande en 2008, puis en Écosse et aux États-Unis, où elle a travaillé comme professeure d'espagnol et serveuse et rencontré de nombreuses difficultés ${ }^{5}$. Elle est ensuite revenue en Espagne et a monté un petit commerce d'artisanat à Carthagène, mais « la hausse de la TVA décidée par le PP et l'absence de 
clients en raison de la crise ", l'ont contrainte selon elle à fermer son petite commerce ${ }^{6}$. Son père, architecte, a été touché par la crise de l'immobilier et s'est retrouvé au chômage. Ce contexte personnel de précarité a déclenché son engagement partisan dès la fondation de Podemos, en créant le cercle local de Carthagène puis en se présentant aux primaires ouvertes qui définirent la liste du parti. Lorsqu'elle devint eurodéputée, elle était encore serveuse saisonnière dans un bar de la ville balnéaire de La Manga del Mar Menor (Murcie). L'eurodéputée Tania González (26 ans) présente un profil assez similaire d'une professeure contractuelle qui a enchaîné les contrats temporaires. Née à Avilés en Asturies "de grands-parents mineurs et de parents professeurs " ${ }^{7}$, elle est diplômée de science politique de l'UCM, débuta un doctorat, mais sans bourse ni financement de thèse, elle retourna en Asturies où elle se prépara au concours de professeur, exerçant durant quatre années en CDD à mi-temps et enchaînant les contrats précaires avant de rejoindre Podemos dès sa naissance en janvier 2014.

Dans le cas des étudiants les plus jeunes, la précarité et l'engagement précoce dans le mouvement étudiant et les organisations de la jeunesse précaire telles que JSF, ont débouché grâce à Podemos sur des accès à la représentation politique sans même avoir eu d'expériences professionnelles préalables, même si ces trajectoires s'expliquent par des situations subies de chômage. Miguel Ardanuy (25 ans), désormais député régional de Madrid, est un autre exemple typique d'un étudiant en science politique de l'UCM qui a fait partie du mouvement étudiant, de JSF, a contribué au 15-M, puis est devenu assistant bénévole en charge des réseaux sociaux lors des européennes dans un groupe dirigé par Eduardo Fernández ( 25 ans). Ce dernier est aujourd'hui député régional de Madrid suivant une trajectoire similaire, du mouvement étudiant, de JSF au 15-M, avant de devenir le responsable de l'équipe "réseaux sociaux » lors des européennes puis lors des municipales à Madrid et des législatives ${ }^{8}$. Dans la même équipe, on trouve aussi Nagua Alba (26 ans), qui a une formation de psychologue de l'éducation mais n'a jamais réellement pu exercer son métier dans de bonnes conditions, se joignant à la campagne de Podemos dès décembre 2013 avant de devenir députée régionale au Pays basque en mai 2015. Clara Serrano (34 ans) était aussi doctorante en philosophie à l'UCM, impliquée dans les mouvements étudiant et féministe puis le 15-M, avant de devenir députée régionale de Podemos à Tolède. Rita Maestre (28 ans) a obtenu un Master en science politique à l'UCM, puis a connu le chômage, milité dans l'association étudiante Contrapoder, JSF et le 15-M en 2011, avant de devenir responsable de la stratégie de campagne de Ahora Madrid lors des municipales puis porte-parole de la mairie après l'élection de Manuela Carmena. Juan Manuel del Olmo (34 ans), désormais député au Congrès de la province de Valladolid suit le même type de trajectoire: diplômé en sciences de l'information à Madrid, il a été indépendant et précaire (déclarant 529 euros bruts par mois en 2013), créant une activité de consultant en nouvelles technologies pour des organisations de l'économie solidaire avant de prendre part au groupe "réseaux sociaux» lors des européennes puis de devenir assistant de Pablo Iglesias. L'ensemble de ces exemples montre bien l'importance de l'engagement de jeunes précaires parmi les nouveaux cadres de Podemos.

\section{De la « Fakul » à Contrapoder : I'université Complutense, un espace clé de socialisation militante}

6 De plus, la faculté de science politique et de sociologie de l'UCM (la « Fakul »), constitue le lieu de socialisation militante par excellence au sein duquel sont passés les cadres les 
plus impliqués du parti. L'immense majorité des membres de son Conseil citoyen national y ont ainsi étudié. La Complutense est une université qui a une longue tradition de formation politique des élites de gauche, mais depuis la fin des années 1990, la «Fakul» a basculé vers une sympathie grandissante pour une gauche plus mouvementiste et alternative, une tendance qui s'est accentuée à travers l'influence croissante en son sein des mouvements étudiants. Au sein même du corps professoral, cela s'est traduit par l'essor d'un nouveau clivage entre les «insiders", le groupe très réduit des maîtres de conférences et des professeurs d'université titulaires, et celui des " outsiders ", l'immense majorité des thésards, professeurs assistants et intérimaires à temps partiel, dont le nombre et la précarité statutaire et économique se sont fortement accrus, les rendant d'autant plus réceptifs aux revendications des étudiants pour l'amélioration des conditions de vie, d'études et d'emploi de la jeunesse. Les situations de convergence objective entre des étudiants protestataires et certains professeurs précaires n'hésitant pas à participer aux grèves, se sont multipliées. Plus qu'un simple centre académique, la Fakul est aussi un espace social et politique où règne les banderoles, les graffitis, les assemblées, les manifestations et les macro-fêtes étudiantes. Ces derniers s'organisent autour de nombreuses associations et les équipes enseignantes doivent tenir compte de leur influence : les interdictions de fumer ou de boire de l'alcool dans les couloirs ne sont pas respectés; une certaine "tolérance " existe à l'égard de la vente de boissons afin de financer des fêtes ou différentes causes ; les tables militantes de mouvements révolutionnaires de gauche (en faveur des prisonniers de l'ETA, de la libération de la Palestine etc.) y trouvent un espace privilégié. La fameuse "moqueta " avant le bar est devenu un espace d'autogestion où fourmillent les collectifs. Des liens amicaux d'affinité et une gestation commune d'idées politiques et de formes d'activisme se sont ainsi créés depuis une dizaine d'années entre ceux qui constituent désormais les principaux cadres de Podemos. La «Fakul»a été le lieu de la confluence entre le travail académique et l'expérience militante de nombreux jeunes madrilènes de gauche issus d'une même classe d'âge.

7 L'association Contrapoder ("Contre-pouvoir») a joué le rôle d'un laboratoire des formes de contestation dès 2006. Son nom reprenait la notion élaborée par Toni Negri dans Le pouvoir constituant, dans lequel il décrit trois phases du "contre-pouvoir", reprises dans le manifeste fondateur de Contrapoder : 1) «la résistance contre le vieux pouvoir, pratiquée dans tous les lieux et secteurs sociaux »;2) «l'insurrection des masses, une fois que ces formes de résistance sont capables de confluer ou d'être articulées dans un noyau dur qui, selon Negri, 'traverse comme une fléche le pouvoir constitué'" ; et 3) «le pouvoir constituant, qui est la capacité de donner une forme institutionnelle aux nouvelles formes de vies qui ont émergé à travers l'insurrection $»^{9}$. Contrapoder naquit à travers un acte fondateur : des étudiants vêtus comme des singes blancs (en écho aux Tute bianche italiennes étudiés par Pablo Iglesias dans sa thèse doctorale) annonçèrent au mégaphone dans la cafétéria leur volonté de «créer un espace de désobéissance dans la faculté $»^{10}$. Íñigo Errejón dirigea l'acte auquel participèrent aussi Pablo Gabandé, Jorge Moruno et Tania González, tous aujourd'hui membres de Podemos. Criant « Deso-be-dien-cia » (« Désobéissance »), ils se dirigèrent ensuite vers un mur de la faculté ou certains dessinèrent la scène de Tommie Smith et John Carlos, poings levés, têtes basses, lors des JO de Mexico en $1968^{11}$. Durant l'action, les militants se filmèrent puis diffusèrent la vidéo sur internet, créant la polémique et illustrant bien leur conscience du pouvoir de l'audiovisuel. À de multiples reprises, le groupe dans le sillage de Contrapoder fut le fer de lance de la contestation au sein de la faculté : il y relaya les 
mobilisations syndicales, se mobilisa contre l'application du plan Bologne, boycotta la présence de représentants de la Banque Santander en 2008, ou encore la venue de Rosa Díez, la dirigeante d'UPyD en mai 2009 puis à nouveau en octobre 2010. Depuis la crise de 2008, la «Fakul» a vu se succèder de nombreuses mobilisations en écho aux mouvements sociaux du reste de l'Espagne: occupations des banques du campus; manifestation féministe seins nus dans sa chapelle le 18 février 2011 pour demander la laïcisation de l'enseignement et dénoncer la "morale patriarcale »; manifestation contre la loi Wert en 2013; relais des trois grèves générales etc. Enfin, il convient d'évoquer que malgré la différence d'âge, un vrai réseau d'amitié s'est créé autour des professeurs Ariel Jerez et Juan Carlos Monedero, impliquant notamment Pablo Iglesias, Íñigo Errejón, Rita Maestre, Irene Montero, Jorge Lago, Luis Alegre ou encore Jesús Montero, qui sont tous désormais membres du groupe dirigeant de Podemos.

\section{Une élite d'entrepreneurs de cause}

8 Un troisième groupe très hétérogène dont sont issus les cadres de Podemos est celui des militants et des activistes du mouvement social qui se sont engagés depuis 2008 dans les mobilisations syndicales anti-austérité, le mouvement des Indignés en 2011, les « marées » verte dans l'éducation et « blanches » dans le secteur de la santé en 2012, les collectifs de jeunes comme JSF et contre les expulsions autour de la Plateforme des affectés par l'hypothèque ( $\mathrm{PAH})$, ou encore les manifestations féministes contre le projet de loi anti-avortement du gouvernement conservateur. L'émergence de Podemos ne peut ainsi pas être comprise sans tenir compte de l'ancrage sociologique antérieur de ses cadres au sein de ces mobilisations sociales anti-austérité, et plus particulièrement, dans le cadre du tissu militant du quartier de Lavapiés à Madrid. Le tout premier local du parti à Lavapiés, au 21 rue Zurita et à l'angle de la rue Torrecilla de Leal, fut d'ailleurs la librairie-coopérative Marabunta de Miguel Urbán, qui devint le quartier général de la nouvelle organisation lors de la campagne des européennes. Lavapiés est non seulement le quartier le plus multiculturel de la ville mais aussi le plus politisé : dans la plupart des bars, locaux ou centres sociaux, des réunions sont organisées et des mouvements sociaux ou groupes politiques emblématiques de l'Espagne contemporaine comme JSF, Democracia Real Ya, Espacio Alternativo ou encore le collectif autogéré autour du Patio Maravillas, s'y sont structurés. Lors de ses premières réunions publiques, Podemos s'est de même appuyé sur la logistique et l'espace fourni par les membres du Teatro del Barrio, à l'angle de la rue, fondé par l'acteur Alberto San Juán, qui est un projet théâtral coopératif, qui donne un espace de libre expression à de nombreux artistes réalistes et à des collectifs qui s'inscrivent pleinement dans la réalité sociale de la crise espagnole. Au sein de Podemos, on retrouve ainsi le codirigeant de la PAH (Rafael Mayoral), des syndicalistes (Sergio Pascual, Diego Cañamero, Jesús Montero), des anciens dirigeants de JSF et figures actives du mouvement des indignés (Miguel Bermejo, Miguel Ardanuy), des féministes et militantes de la "marée violette» (Beatriz Gimeno, Clara Serra, Paula Baeza), des leaders de Contrapoder (Errejón et Iglesias) et du mouvement étudiant (Ramón Espinar, Rita Maestre), de nombreux professeurs, fonctionnaires territoriaux et personnels de la santé. Le noyau des fondateurs et des cadres dirigeants de Podemos se trouve donc ancré dans un territoire urbain madrilène, et se fonde sur la confluence d'intellectuels, d'activistes du mouvement social et de membres du mouvement des indignés. 


\section{Izquierda Anticapitalista et l'importance d'une structure organisationnelle}

Enfin, un dernier groupe doit être évoqué dans la mesure où il a permis de constituer l'ossature initiale de certains « cercles » locaux dès le printemps 2014 : il s'agit des 600 militants environ d'Izquierda Anticapitalista (IA) qui ont joué un rôle clé dans la campagne des européennes, en faisant bénéficier Podemos de ses structures organisationnelles et de la forte mobilisation de ses militants. Tant sa branche madrilène que son collectif catalan Revolta Global (« Révolte globale »), lui permirent de disposer d'un petit groupe de militants très fortement impliqués sur le terrain. En outre, ce fut à l'initiative de membres d'IA que fut rédigé le manifeste préalable à la fondation de Podemos. IA est un parti d'extrême-gauche fondé en 2008 par l'organisation Espacio Alternativo («Espace alternatif») qui fit scission d'Izquierda Unida $^{12}$. Teresa Rodríguez, ex-eurodéputée et désormais députée régionale et dirigeante du parti en Andalousie, Miguel Urbán ou encore Luis Alegre, furent tous membres d'IA jusqu'à sa dissolution en janvier 2015 pour que ces membres puissent se présenter à des responsabilités au sein de Podemos ${ }^{13}$. Dès sa naissance, les militants d'IA, emmenés par Miguel Urbán et Teresa Rodríguez, investirent massivement le parti, ce qui fut rapidement perçu comme une menace, notamment par Íñigo Errejón, partisan de la "transversalité ", mais aussi d'une certaine manière par Pablo Iglesias, qui ne voyait pas d'un très bon œil les tentatives d'entrisme et de prise de contrôle du parti par les militants d'extrême-gauche. On constate aujourd'hui une très bonne insertion des ex-militants d'IA au sein de Podemos. Nombreux sont ceux qui ont accédé à des responsabilités internes ou à des mandats électifs. À Madrid, Pablo Iglesias a d'ailleurs cherché à s'allier avec eux autour de la candidature de Ramón Espinar pour contrer l'influence grandissante des partisans d'une posture plus conciliante à l'égard du PSOE autour d'Íñigo Errejón, de Rita Maestre et de José Manuel López. Lors du second Congrès de Vistalegre en février 2017, les anticapitalistes obtinrent seulement $10 \%$ des voix et deux conseillers sur les 62 du Conseil citoyen national. Mais même s'ils défendent des positions plus radicales que celles d'Iglesias, ils demeurent des alliés objectifs face au principal courant de l'opposition interne représenté par Íñigo Errejón.

\section{Une « stratégie populiste » pour faire émerger une nouvelle opposition politique}

10 Pour Pierre-André Taguieff, le populisme caractériserait «un style politique », "un ensemble d'opérations rhétoriques » qui dépendrait étroitement « des appels au peuple et de l'exploitation symbolique de certaines représentations sociales $»^{14}$. Il dégage un ensemble de traits communs aux "mouvements populistes»: "crise de légitimité politique préalable »; « appel au peuple contre les élites au pouvoir »; « rejet de toute médiation politique»; "phénomène transitoire et instable»; et "absence d'une idéologie revendiquée ». Notons d'abord que ces tendances sont discutables. De plus, la " crise de légitimité politique » caractérise le contexte général et non le style discursif de Podemos. Le parti ne rejette pas non plus la médiation politique, puisqu'il s'est luimême structuré rapidement comme une organisation partisane assez classique, accepte les règles du jeu électoral, s'inscrit dans le champ politique légitime et cherche à accroître son influence au sein des institutions. Le fait qu'il doive gouverner en 
coalition au sein de plusieurs villes majeures (Madrid, Barcelone, Cadix) dans les années à venir réfute aussi l'idée d'un " phénomène instable et transitoire » qui serait amené à disparaître. Son idéologie à mi-chemin entre les références traditionnelles du communisme et du socialisme, contredit aussi l'idée d'une absence d'idéologie. Même s'il adopte un discours transversal en cherchant à s'adresser à une majorité des citoyens, Podemos est un parti de gauche et demeure perçu comme tel par les électeurs. Il resterait donc uniquement «l'appel au peuple» pour l'associer au "populisme ", une preuve bien maigre. Dans cette partie, je montre que le concept de populisme est trop réducteur pour comprendre la nature du parti, même s'il est possible de parler d'une "stratégie populiste " d'accès à la représentation politique à travers la genèse d'un mouvement oppositionnel nouveau.

\section{Appel au peuple et défense des classes populaires}

Il est vrai que les discours de campagne du parti sont imprégnés d'oppositions facilement intelligibles, qui articulent souvent des visions du monde autour d'une série de dichotomies : « les gens honnêtes/les élites corrompues »; « les citoyens ordinaires/ la caste »; « ceux d'en bas/ceux d'en haut »; « les petites gens touchées par la crise/les banquiers sans scrupule"; "la nouvelle politique/la vieille politique»; "le sens commun/l'idéologie »; "le pays réel/le pays des élites»; «la majorité sociale/la minorité des privilégiés » etc. Cette volonté de "dichotomiser l'espace politique ${ }^{15}$ est souvent retenue comme typique du populisme. La volonté de créer un mouvement populaire s'appuyant sur des mécanismes de participation malgré l'«hyperleadership " du secrétaire général est aussi mise en avant en analogie avec le " chavisme ». Mais il existe un fossé important entre les discours « de campagne » et «institutionnels » du parti ${ }^{16}:$ si les premiers laissent souvent apparaître un style discursif qui s'apparente à un "populisme de gauche", les seconds sont plus proches des discours classiques d'opposition d'un parti socialiste. C'est par exemple le cas du concept de "caste » qui n'est plus utilisé par les dirigeants dans leurs conférences de presse et déclarations quotidiennes. Là où, lors des campagnes, des "chaînes d'équivalence " pour reprendre le concept d'Ernesto $\mathrm{Laclau}^{17}$, sont formulées entre des acteurs ou des processus distincts, dont les actions et intérêts sont présentés comme convergents, ce type de processus discursif "populiste » est rarement utilisé dans les discours institutionnels : un autre exemple est celui du traitement différencié du PSOE, vivement critiqué en campagne, mais présenté comme un allié nécessaire pour former des majorités alternatives dans les discours institutionnels. Podemos propose donc clairement un discours à géométrie variable typique des partis contestataires qui veulent parallèlement jouer le rôle de partis "pivots » et partenaires potentiels de coalition : pour reprendre la distinction classique de Giovanni Sartori, il cherche à concilier un "potentiel de chantage» pour influer sur les stratégies de compétition des autres partis, avec un "potentiel de coalition" propre aux partis mineurs à vocation gouvernementale ${ }^{18} ;$ il recourt donc tantôt à une stratégie « d'opposition de principe » à travers l'usage d'une stratégie discursive populiste, mais en s'appuyant aussi sur une stratégie "d'opposition classique $»^{19}$. Le populisme peut donc certes qualifier le style discursif du parti en campagne, mais pas la nature même du parti ou celle de l'ensemble de ses discours.

De plus, au-delà de la rhétorique de l'appel au peuple lors des campagnes, Podemos a su convaincre une partie importante des secteurs sociaux les plus affectés par la crise, 
faisant d'abord de lui un parti « populaire » du point de vue de la sociologie électorale : lors des législatives de 2015, ses principaux soutiens étaient les jeunes précaires, mais il attirait autant que le PSOE les secteurs appauvris de la classe ouvrière et des classes moyennes ${ }^{20}$. Le fait qu'une partie importante des classes populaires se détournent du PSOE et soient séduites par son discours plus direct traduit une forme de radicalisation des électeurs de gauche. Les enquêtes postélectorales montrent que sa progression s'est surtout effectuée au détriment d'IU lors des européennes de 2014 puis du PSOE en 2015, tout en remobilisant certains abstentionnistes "dans le jeu» et en attirant de nouveaux jeunes électeurs ${ }^{21}$. Comme ailleurs en Europe, le retour d'un style "plébéien » d'élites qui cherchent à recomposer le jeu politique illustre une forme de « ras-le-bol » des petites gens face aux promesses non tenues et à la médiocrité de «sortants" qui pensent parfois plus à conserver leurs postes qu'à améliorer concrètement la vie des citoyens. Alexandre Dorna considère que « lorsque les masses populaires sont exclues longtemps de l'arène politique, le populisme, loin d'être un obstacle, est une garantie de démocratie, car il évite que celle-ci se transforme en pure gestion $»^{22}$. Dans un article intitulé «Podemos: le parti des classes populaires », Pablo Iglesias évoque clairement cette dimension visant à articuler un " discours âpre » qui puisse séduire les classes populaires mais aussi le peuple espagnol dans son ensemble en jouant sur la crise de la représentation :

«Pendant longtemps, on nous associera avec des notions telles que la 'régénération', la 'nouveauté' et la 'jeunesse', mais notre terrain principal doit être celui qui fait de nous la force politique de référence de classes populaires en expansion comme résultat de l'appauvrissement d'une bonne partie des classes moyennes (travailleurs du secteur public, indépendants, petits entrepreneurs et artisans), du précariat, de la jeunesse et de la classe ouvrière. C'est pour cela que nous devons être une force avec un discours âpre et de classe et avec un style plébéien, qui doit même être visible dans notre manière distincte de nous habiller. Podemos doit être le parti des classes populaires, ce doit être le 'parti du peuple' au sens large et littéral du terme, puisque la gauche traditionnelle, en devenant plus gestionnaire que populaire, a d'une certaine manière renoncé à cet électorat ${ }^{23}$.

\section{Politiser les questions de société par une posture transversale}

Podemos ne cherche donc pas tant à «manipuler la réalité politique et la dramatiser à l'extrême $»^{24}$ comme l'évoque Patrick Charaudeau à propos du populisme, qu'à " politiser la douleur ${ }^{25}$, à dénoncer les injustices sociales dans une stratégie en fait assez classique d'opposition et de repolitisation des enjeux de société qui touchent à des aspects concrets de la vie des citoyens (droit au logement, à la liberté d'expression, à l'éducation et la santé etc.). Il cherche à allier un discours émotionnel en campagne, et rationnel face à ses adversaires dans les débats publics et télévisés, par un intense travail de préparation, de recherche de données chiffrées, de petites formules et de slogans, souvent repris par les grands médias et déstabilisant des adversaires qui s'étaient habitués à la "langue de bois ». Une autre clé de son succès est ainsi un discours s'adressant à la majorité, qui est différent et beaucoup plus transversal que celui de la gauche alternative traditionnelle, comme l'explique Pablo Iglesias lui-même : «J'ai vu que ce discours fonctionnait et qu'il plaisait. Les gens m'arrêtaient dans la rue et me disaient : 'Je sais que tu es de gauche et je ne le suis pas, mais je suis d'accord avec ce que tu dis' $»^{26}$. Il fonde son succès relatif sur un travail idéologique et intellectuel 
délaissé par les autres partis de gauche. Ce qui le distingue d'IU et de l'extrême-gauche traditionnelle est de chercher à convaincre l'ensemble de l'opinion publique.

Il ne veut pas simplement apparaître comme un parti critique "d'opposition », mais dit et redit sa volonté de gouverner dans les villes, les régions et au niveau national afin de mettre en pratique des politiques alternatives. Il cherche à allier «l'éthique de conviction » et « l'éthique de responsabilité $~^{27}$, ce qui génère de nombreuses difficultés et tensions en interne. Il est donc beaucoup plus pragmatique qu'IU ou le Parti de gauche en France, critique allégrement le PSOE en campagne électorale, mais n'a pas hésité à s'allier avec lui ensuite pour gouverner à Valence, Barcelone, Madrid, SaintJacques de Compostelle ou à La Corogne. Ses dirigeants cherchent stratégiquement à se démarquer des étiquettes traditionnelles et du clivage gauche-droite. Le choix du logo très épuré du parti, trois cercles superposés, et la couleur assez neutre choisie par la formation, le violet, mélange de rouge et de bleu, à la confluence des couleurs traditionnelles de la gauche et de la droite, ou encore le slogan très généraliste (« Oui, nous pouvons »), sont autant d'éléments qui illustrent bien cette volonté de proposer l'image d'un parti ouvert, nouveau et progressiste. En refusant d'être "étiqueté " comme un parti «de gauche " malgré l'évidence de ses références idéologiques, il se place dans une posture visant à articuler un discours plus transversal dirigé à tous. Certains y verront une marque de populisme, mais ceci constitue aussi une caractéristique des partis « attrape-tout », comme l'illustre En Marche! en France, plus axés sur la conquête du pouvoir et la volonté de convaincre une majorité des citoyens que sur la défense acharnée d'une idéologie ${ }^{28}$. Son insertion dans le groupe de la gauche unitaire au Parlement européen ne laisse cependant aucun doute sur sa proximité politique avec la gauche alternative ${ }^{29}$. Mais contrairement à Syriza, qui n'a jamais cherché à occulter son idéologie héritée de l'eurocommunisme, Podemos évite les labels d'un parti "communiste » ou de "gauche radicale ", jugés trop péjoratifs, et préfère celui d'un « mouvement citoyen ». Il est d'ailleurs perçu sans ambiguïté comme "de gauche »: sur une échelle gauche-droite de 0 à 10, les Espagnols le situent à 2,5, soit au cœur de la gauche, voire même plus proche de la "deuxième gauche ", situant le PSOE à 4 , plus au centre-gauche ${ }^{30}$.

\section{Un programme politique « socialiste hétérodoxe »}

Le populisme n'est pas une idéologie, mais ce que beaucoup disent dans leurs interprétations du populisme est qu'il se caractériserait par l'association de contenus idéologiques contradictoires. Pensons par exemple au Front National qui associe des mesures radicales et ultra-conservatrices sur l'immigration et la lutte contre la délinquance autour des valeurs d'ordre et d'autorité, avec des propositions socialisantes (augmentation du Smic, nationalisation des autoroutes par exemple) ${ }^{31}$. Une analyse détaillée du programme politique de Podemos, montre cependant qu'il a rapidement modéré son radicalisme initial, incarnant aujourd'hui un "socialisme hétérodoxe" au sens où il diffère des positions plus au centre-gauche des partis sociaux-démocrates dominants mais en crise aujourd'hui en Europe. Le "Programme économique pour les gens $\aleph^{32}$ présenté en 2014 pour les européennes s'inspirait des recommandations des économistes Juan Torres et Vicenç Navarro, en cherchant à se faire le porte-parole des idées issues des mouvements sociaux : il prévoyait un plan de relance dans la dépendance, la recherche et les énergies renouvelables pour diversifier l'économie espagnole, d'abroger la loi travail votée par le PP, de diminuer le temps de 
travail, de mettre fin aux coupes budgétaires dans la santé et l'éducation, ou encore d'adopter un moratoire sur les expulsions. Ce qui favorisa son " procès en populisme ", furent un ensemble de propositions socialisantes (retraite à 60 ans; nationalisation des compagnies d'électricité; rente basique universelle) et anti-conventionnelles (référendum sur la monarchie; moratoire sur le paiement de la dette publique). Il est clair que ces mesures donnaient au Podemos des débuts un profil plus proche des "gauches radicales ». Mais il a modéré son programme dans sa quête de pouvoir: il n'évoque plus les nationalisations, la rente basique ou le référendum sur la monarchie et propose désormais une renégociation de la dette. Il s'agissait plus d'effets d'annonce pour permettre à un groupe ultra-minoritaire d'entrer dans un espace politique compétitif et fermé, en empiétant sur l'espace traditionnel d'IU.

Lors des municipales de 2015, Podemos s'effaça au profit de plateformes citoyennes locales incluant un ensemble plus important d'acteurs. Une mesure emblématique mise en avant presque partout fut la proposition de « lois de comptes ouverts » pour obliger les municipalités à rendre public leurs choix budgétaires pour une plus grande transparence. Mais les équipes de campagne défendirent surtout des propositions spécifiques ancrées dans les problématiques locales: Barcelona en Comú, la lutte contre les appartements touristiques illégaux qui suscitent des nuisances et un malaise croissant des barcelonais; Cadiz Sí Se Puede, les mesures d' urgence sociale" (moratoire sur les expulsions, revenu minimum d'insertion) prônées par Podemos, dans une province où presque $30 \%$ de la population active est sans emploi ; Valencia en Comú axa sa campagne sur « la fin du règne de la corruption » dans une ville qui a connu de nombreux scandales (Gürtel, Imelsa, Taula etc.) et le règne de Rita Barberá et du PP depuis 1991; enfin, En Marea en Galice insista sur l'idée de redonner le pouvoir aux citoyens, de défendre les intérêts régionaux et de reprendre le pouvoir au PP. Les plateformes municipales soutenues par Podemos illustrèrent ainsi des alliances à géométries variables, fortement ancrées dans les réalités des villes et cherchant à impliquer des acteurs issus de la société civile, ce qui s'avéra souvent un franc succès.

Enfin, son programme économique des législatives de décembre 2015 montre bien cette forme de "socialisme hétérodoxe": revenu minimum d'insertion de 600 euros par mois ; prestation complémentaire jusqu'à 900 euros pour les bas salaires ; augmentation du salaire minimum de 640 à 800 euros; réforme fiscale et plan de lutte contre la fraude; conditionnement de la réduction du déficit public à $3 \%$ du PIB à la diminution du chômage; plan de "développement productif» dans l'économie verte, la réhabilitation des immeubles, les nouvelles technologies et la recherche ${ }^{33}$. Des mesures telles que le plan de développement productif, la réforme fiscale, le revenu minimum d'insertion et la prestation complémentaire pour les travailleurs précaires pourraient être adoptées par la plupart des partis sociaux-démocrates européens. L'augmentation $\mathrm{du}$ salaire minimum constitue aussi historiquement une mesure soutenue par la gauche. La diminution de l'âge de départ à la retraite de 67 à 63 ans va il est vrai à rebours de la tendance inverse en Europe. Le conditionnement de la réduction du déficit public à celle du chômage, et le fait d'« étudier la possibilité » de renégocier la dette publique "en accord avec les créanciers de l'Espagne " (la formulation est ellemême très prudente), illustrent une convergence idéologique avec d'autres partis de la gauche alternative en Europe du sud, tels que Syriza en Grèce et le Bloco de Esquerda au Portugal. Podemos propose ainsi un programme socialiste que Pablo Iglesias compare « au socialisme mitterrandien du programme commun avant le tournant de la 
rigueur en $1983 »^{34}$. Ce socialisme hétérodoxe s'observe clairement du point de vue de la pression fiscale : augmentation progressive du taux marginal de l'impôt sur le revenu (45\% actuellement) pour les revenus supérieurs à 5000 euros par mois, jusqu'à atteindre $55 \%$ pour les revenus de plus de 25000 euros par mois ; impôt de $25 \%$ sur les articles de luxe ; impôt sur les énergies non renouvelables (hydrocarbures, charbon) ${ }^{35}$; diminution du seuil d'entrée de l'impôt sur le patrimoine (de 700000 euros actuellement à 400 000).

Il suggéra aussi de réformer l'impôt sur les sociétés en fonction de la destination des bénéfices, en augmentant la pression fiscale sur les bénéfices distribués aux actionnaires tout en la diminuant pour ceux redistribués en faveur d'investissements productifs au sein de l'entreprise. Son programme plaida pour «renforcer le soutien aux petites et moyennes entreprises ", notamment en "diminuant la pression fiscale les concernant", et en "créant une banque publique dont l'objectif serait d'offrir des prêts, sur la base de critères sociaux et stratégiques, afin d'appuyer la création d'entreprises et de start-ups ${ }^{36}$. Il s'agirait aussi de «faciliter l'affiliation à la Sécurité sociale des travailleurs indépendants ». Podemos proposa de plus de mettre en place un «taux super-réduit » de TVA à $4 \%$ sur les aliments de base et les boissons non alcoolisées, de même que d'appliquer le "taux réduit " de 10\% (plutôt que 21\% aujourd'hui) à l'ensemble des ressources de base (chauffage, gaz, eau, électricité). L'influence des mouvements sociaux et surtout des revendications de la Plateforme des affectés par l'hypothèque fut aussi patente à travers sa "Loi 25 d'urgence sociale ", en référence à l'article 25 de la déclaration des droits de l'homme, qui propose "d'interdire les expulsions sans alternative de relogement», de "limiter la responsabilité du particulier endetté au bien concerné par le prêt immobilier », et d'instaurer « le droit à la donation pour paiement " permettant aux particuliers de solder leurs dettes bancaires en échange de la cession de leur bien. Il cherche ainsi à donner une réponse politique à l'une des problématiques majeures de la crise espagnole. On note enfin un élargissement progressif et une complexification de ses propositions : le programme des législatives du 26 juin 2016 présentait ainsi plus de 300 mesures sur la dépolitisation de la justice, la lutte contre la corruption, l'égalité femmes-hommes, l'écologie etc.

\section{Une organisation interne sous tension : du mouvement citoyen au parti traditionnel}

L'enjeu de l'institutionnalisation partisane est la troisième dimension centrale à évoquer dans la mesure où Podemos est d'une certaine manière " un parti qui ne veut pas en être un ». Il est né en se présentant comme un mouvement citoyen fondé sur des assemblées de rues autogérées, où ses sympathisants se réunissaient initialement de façon hebdomadaire dans des espaces publics ouverts, dans l'esprit de l'occupation des places par le mouvement des indignés. Encore aujourd'hui, les réunions de ses "cercles» locaux sont annoncées sur les réseaux sociaux ou en utilisant Facebook. Podemos est d'abord un parti 2.0 et d'électeurs. Lors des européennes, il utilisa le financement participatif («crowdfunding»), tout en publiant le détail de ses dépenses sur internet. Ses cinq eurodéputés élus se sont engagés à ne percevoir que 2225 euros nets (soit trois fois le salaire minimum en Espagne) et à redistribuer le reste de leur salaire (6400 euros) à des organisations de leur choix impliquées dans le mouvement 
social. Le parti a étendu cette pratique à l'ensemble de ses élus. Même s'il existe un manque de contrôle sur le respect réel de cette obligation interne, et que les dons peuvent donner lieu à des conflits d'intérêt, ses élus signent des déclarations de patrimoine et nombreux sont ceux qui publient le détail de l'utilisation de leur rémunération sur internet. Malgré les critiques possibles, force est toutefois de constater que Podemos tente d'aller beaucoup plus loin que les autres partis dans l'incitation à la limitation des salaires et de pratiques plus transparentes. Dans cette partie, je synthétise les aspects principaux de son organisation interne, en montrant la volonté d'instaurer de nouvelles pratiques, d'utiliser les réseaux sociaux pour faire campagne mais aussi pour s'organiser, de se structurer comme un mouvement d'assemblées autogérées, même si l'institutionnalisation a aussi engendré un renforcement de la centralisation autour du secrétaire général.

\section{L'usage des réseaux sociaux et de l'internet : un parti 2.0 ?}

Podemos est un parti en pointe sur l'usage des réseaux sociaux et de l'internet, tant dans son fonctionnement interne, que pour faire campagne. À certains égards, sa campagne des européennes rappela l'avantage technologique pris par Barack Obama sur ses concurrents en 2008. La manière même d'élaborer son programme fut très innovante : il s'agissait d'un Programme participatif avec des propositions formulées par le groupe des fondateurs puis proposées à la discussion sur les réseaux sociaux. Les citoyens pouvaient voter en approuvant ou non les propositions et suggérer des amendements. Contrairement à la pratique des partis traditionnels, qui engendre l'imposition du programme électoral par les élites dirigeantes sans discussions ni débats avec les militants (et encore moins avec les citoyens), la définition interactive du programme électoral suscita un fort engouement et permit à Podemos d'impliquer les gens autour de son projet et de se créer une audience. Le succès de l'initiative fut tel qu'il obligea d'ailleurs les autres partis à réagir. Le Parti populaire créa rapidement une plateforme internet, "En 140, l'Europe avance», incitant les citoyens a envoyé des propositions de 140 caractères suivant le format d'écriture de Twitter ${ }^{37}$. Equo, petit parti écologiste, proposa un Wikiprogramme. IU développa de même un «Programme ouvert » aux suggestions de ses militants : comme dans le cas de Podemos, il s'agissait pour les internautes de proposer des amendements à un programme prédéfini, mais seules les personnes affiliées au parti pouvaient participer et non l'ensemble des citoyens. Mais le Parti socialiste n'a pas réagi à cette tendance: seule l'une de ses fédérations locales, le parti socialiste de Galice (PSdG) a proposé une initiative de ce type, «Réinventons la Corogne » lors des municipales postérieures de mai 2015. Lors de ces recueils virtuels de doléances, les questions du chômage et du futur de la jeunesse émergèrent très clairement comme les principales préoccupations des citoyens. La définition interactive du programme de Podemos ouvrit d'une certaine manière une nouvelle séquence politique, caractérisée par la réappropriation active par les autres partis d'innovations politiques proposées initialement par le parti violet (slogans, pratiques, propositions etc.).

21 Podemos utilisa aussi très bien les réseaux sociaux. Une équipe de 15 étudiants et jeunes bénévoles administrèrent les différentes adresses internet et comptes du parti sur les réseaux sociaux durant la campagne. Chaque jour, ils débattaient durant plusieurs heures sur le sujet d'actualité ou les déclarations polémiques de leurs opposants qui pouvaient être les plus vus, avant de lancer la tendance par des messages 
ciblés. À plusieurs reprises, l'une des étiquettes mises en avant par l'équipe de Podemos sur les réseaux sociaux constitua le thème le plus débattu du moment. Certains messages sur la page Facebook du parti ont été partagés par plus de 2000 personnes ce qui, selon certains analystes, a pu l'amener à atteindre jusqu'à 750000 personnes durant la campagne ${ }^{38}$. D'autres spécialistes des réseaux sociaux ont même estimé que Podemos peut avoir réussi à contacter environ deux millions d'Espagnols ${ }^{39}$. Le succès des outils virtuels du parti a ensuite triplé dès l'annonce des résultats le soir du 25 mai 2014 : 48h après les européennes, Podemos comptait 194000 suiveurs sur Twitter, 380 000 sur Facebook, et Pablo Iglesias était devenu le dirigeant politique le plus suivi sur Twitter avec 322000 «followers", illustrant un véritable "phénomène médiatique » ${ }^{40}$. Rita Maestre, désormais porte-parole de la mairie de Madrid, résume bien la clé de ce succès digital, en expliquant que les forces vives de Podemos, largement impliquées dans le 15-M et les mouvements sociaux, maitrisent mieux ces outils que les militants d'autres partis : «Nous fonctionnons en réseau de façon naturelle. Notre préparation est très bonne, nous n'avons pas besoin de former ou d'avoir recours à des personnes extérieures pour diffuser nos messages. Nos militants sont déjà à la pointe des nouvelles technologies $~^{41}$. L'internet et les réseaux sociaux auraient été utilisés comme "des espaces d'interaction", en faisant l'effort "de dialoguer avec les gens, de répondre aux tweets et de créer un espace d'échange ", contrairement aux autres partis qui se limitent souvent à diffuser leurs messages, en reproduisant la tendance oligarchique de l'imposition du contenu par le haut sans débat ${ }^{42}$. L'adepte de Twitter pouvait ainsi déceler les manœuvres purement stratégiques des grands partis, recrutant des collaborateurs pour faire vivre les comptes de leurs candidats : la tête de liste du PSOE, Elena Valenciano, revint sur le réseau le 20 décembre 2013 après avoir été inactive pendant 10 mois et publia seulement quelques photos durant la campagne ; Miguel Arias Cañete, ministre de l'agriculture et tête de liste du PP publia deux ou trois tweets par jour, après avoir créé son profil juste avant le début de la campagne. Depuis lors, tant Valenciano que Cañete ne font plus vivre leurs comptes Twitter. À l'inverse, le profil de Pablo Iglesias est actif depuis 2011, et il l'utilise lui-même quotidiennement. Tant par son programme participatif que par son utilisation des réseaux sociaux, Podemos marqua la campagne, fut à la pointe des innovations technologiques et obligea les autres partis à s'adapter.

Podemos a d'ailleurs aussi recours aux nouvelles technologies de l'information et de la communication, notamment aux applications de vote électronique, dans son fonctionnement organisationnel interne. Ainsi, par exemple, le dimanche 19 octobre 2014 dans la soirée, les 26 membres de l'équipe technique chargés d'organiser l'assemblée constituante se connectèrent sur Telegram pour un « rendez-vous virtuel». Grâce à cette application de téléphone portable, ils débattirent et votèrent la méthode de choix des documents fondateurs du parti. Cet épisode fut illustrateur du fait que l'utilisation des nouvelles technologies constitue l'une des pierres angulaires de l'organisation interne de la formation ${ }^{43}$. Dès son lancement le 17 janvier 2014, Twitter a dû suspendre deux fois le compte de Podemos devant l'avalanche de "Tweets" des suiveurs. Depuis les européennes, Podemos a formé trois groupes de travail, « Réseaux sociaux ", " participation » et " technologies ", qui diffusent du contenu sur la toile et les réseaux sociaux. Ce groupe se fonde notamment sur des militants de Juventud Sin Futuro, qui ont acquis des compétences sur le partage numérique des informations dans le cadre du mouvement des indignés. Durant la campagne des européennes, Eduardo Rubiño géra le groupe " réseaux sociaux » et coordonna une équipe de 15 à 20 
volontaires. Ce travail d'organisation et de diffusion de contenus numériques a d'ores et déjà porté ses fruits. En 2015, Podemos était ainsi la formation politique la plus suivie sur Twitter (428 000 suiveurs contre 181000 pour le PSOE et 177000 pour le PP), et dominait encore plus ses concurrents sur Facebook (856 000 suiveurs contre 77000 pour le PP et 73000 pour le PSOE). On constate ainsi aisément que le parti cherche à exploiter une «brèche » digitale et générationnelle par rapport à ses concurrents. La jeunesse d'un nombre important de ses militants constitue un atout majeur. Comme le déclare Fernando, un militant du cercle de Lavapiés de 33 ans, qui se dit «journaliste précaire » et « hyperconnecté » sur Twitter, Facebook et Instagram :

«Nous avons un coup d'avance sur les autres partis. Nous sommes mieux organisés qu'eux sur les réseaux sociaux et internet. Il y a un vrai travail d'organisation. Je pense que cela va payer et faire la différence. On voit d'ailleurs les dirigeants du PP qui commencent à dire qu'ils doivent réagir et être plus actifs. On est en train de créer des brèches dans les partis traditionnels. L'idée est d'attirer les forces vives, de faire travailler les jeunes créateurs, de s'entourer d'ingénieurs et de professionnels de l'internet. Podemos est une sorte de 'start-up' politique où se retrouve ceux qui veulent mettre l'innovation au service du changement politique $»^{44}$.

En octobre 2015, Pablo Iglesias était désormais suivi par 663000 personnes sur Twitter contre 614000 pour Mariano Rajoy, et très loin devant Pedro Sánchez (96 000). À cela, il faut ajouter les dizaines de comptes sectoriels et régionaux promus par les cercles et les assemblées territoriales du parti, mais aussi ceux animés par les Espagnols à l'étranger. Grâce l'application Telegram, des débats en temps réel pouvant inclure simultanément des représentants de près de 250 cercles distincts ont lieu lors des grandes réunions publiques du parti. La progression des adhésions sur les réseaux sociaux s'est d'ailleurs très fortement amplifiée après les européennes qui ont marqué un point d'inflexion. Àngel, un étudiant de 25 ans impliqué au sein du groupe "Participation » dirigé par Miguel Ardanuy, m'expliqua que le parti utilise aussi l'application de vote Appgree, disponible sur les téléphones portables, qui permet, à partir de critères sémantiques et numériques, de sélectionner parmi les centaines d'opinions et de propositions des utilisateurs sur un sujet donné, lesquelles présentent les plus forts taux d'interaction, de popularité et de redondance. Pour lui, il s'agirait d'un "exemple d'intelligence collective " permettant de faire remonter vers la direction du parti les positions les plus appréciées par les sympathisants de base ${ }^{45}$. Cela permet aussi, grâce aux technologies de l'information, de tirer profit et de structurer le travail de processus d'assemblées qui ont montré leurs limites organisationnelles suite au mouvement des indignés. L'application Appgree a ainsi été utilisée lors de l'assemblée citoyenne d'octobre 2014 pour sélectionner 50 questions (sur 15 000) émanant des cercles de base à travers 260000 votes, qui furent ensuite posées aux porte-paroles de chaque aire thématique. Enfin, en utilisant la technologie américaine Reddit, la formation a créé «Plaza Podemos », un site internet d'agrégation et d'interaction entre utilisateurs, qui permet de faire vivre ensemble les critiques de certains secteurs, les propositions et les débats en jouant le rôle d'une plateforme de contenu.

\section{Autogestion, horizontalité et démocratie participative : les produits d'appel de Podemos}

24 De plus, au-delà de cet usage innovant des nouvelles technologies, l'autogestion, l'horizontalité et la démocratie participative constituèrent les principaux produits 
d'appel initiaux de Podemos. Ses structures de base sont constituées par des assemblées ou « cercles » en s'inspirant de la dynamique assembléiste du mouvement des indignés. La dynamique d'auto-organisation s'est propagée avec un fort engouement après les européennes dès juin 2014 : en moins d'un an, environ 1000 "assemblées citoyennes » (dont une cinquantaine rien qu'à Madrid) ont été constituées sur l'ensemble du territoire, créant de toute pièce un réseau qui comptait déjà 260000 sympathisants début 2015. Cherchant à s'appuyer sur une large base sociale, le parti remet en cause la division traditionnelle entre les " militants » (qui paient normalement une cotisation) et les "sympathisants »: il se structure de fait comme un parti d'électeurs où le militantisme est plus fluide. Pour être considéré comme "sympathisant» (ce qui implique selon ses propres critères, faire partie de l'organisation), il suffit de s'affilier à l'un de ses cercles locaux ou de s'inscrire sur le site internet du parti. Aucune cotisation n'est exigée, mais les dons sont encouragés. Un groupe local peut naître à l'initiative de seulement deux personnes, dans un village, un quartier ou une ville. Il peut se développer ou se dissoudre suivant la volonté de ses membres, comme un réseau de suiveurs sur Twitter ou Facebook, ce qui donne corps à un militantisme très diffus et quasi-entièrement autogéré. Une assemblée doit transmettre à la direction nationale les informations relatives à la dénomination du groupe, aux noms, prénoms et aux contacts courriels de ses membres avant d'entrer officiellement en vigueur. L'ensemble des membres des assemblées locales peuvent prendre part aux votes virtuels ou présentiels des grandes décisions nationales, mais aussi participer aux assemblées municipales et régionales et au Congrès du parti qui doit avoir lieu tous les trois ans selon ses statuts. Les réseaux sociaux jouèrent donc un rôle central dans la phase initiale de constitution du parti dans la mesure où il ne disposait que de très peu de locaux sur le terrain, ce qui eut pour avantage de limiter les besoins de financement. Podemos s'organise à travers un système de participation ouvert, puisque des personnes non affiliées peuvent participer à ses assemblées. Ce fonctionnement en réseau séduit beaucoup les jeunes générations.

Toutefois, avec le temps, l'auditoire des réunions des cercles a eu tendance à se restreindre aux militants, l'organisation des délibérations devenant de même plus organisée, avec des temps de parole et une durée limitée des réunions ${ }^{46}$. Mais suivant l'esprit des assemblées du $15-\mathrm{M}$, les décisions organisationnelles routinières sont le plus souvent prises à l'unanimité. Il n'y a pas de porte-parole, chacun peut intervenir, même si les responsables des commissions thématiques, et surtout désormais, les élus locaux, régionaux ou nationaux lorsqu'ils y participent, jouent un rôle déterminant. Au départ, certains militants voulaient clairement interdire les porte-paroles pour éviter le personnalisme et marquer le rejet de la démocratie représentative au profit de la démocratie directe: mais pour une question d'efficacité, il a été décidé que des représentants des cercles locaux seraient élus par l'ensemble de leurs membres afin de défendre leurs revendications aux niveaux régional et national. Refusant d'apparaître comme un parti de " délégués ", à la structuration proche des partis traditionnels, force est donc de constater que des pratiques de délégation du pouvoir se sont peu à peu imposées afin de faciliter le travail de coordination entre les différents échelons du parti. Certains militants sont ainsi assez critiques de la remise en cause rapide du modèle d'un parti plus horizontal et de l'affaiblissement du rôle des cercles. Pablo Echenique, le secrétaire d'organisation, considéra lui-même à l'automne 2016 qu'« il faut réactiver les cercles, puisque nombreux sont ceux qui ne sont plus actifs $»^{47}$. À Valence, j'ai rencontré certains sympathisants déçus, pour qui la primauté initiale de la 
démocratie directe à la base et du rôle des cercles aurait été remise en cause par l'institutionnalisation des conseils citoyens. Rencontrée au quartier général du parti rue Turia, Lidia, une commerçante de la vieille ville qui milite dans le groupe local estime ainsi que «les cercles ne jouent quasiment plus aucun rôle, à part dans les quartiers. Maintenant, ce sont les réunions du conseil citoyen de la ville qui sont déterminantes. Les cercles servent surtout comme outils de mobilisation lors des campagnes, mais le lieu de la délibération et de la décision est devenu le conseil citoyen $»^{48}$.

Il faut donc conclure par deux remarques. D'une part, même si le fonctionnement des cercles s'inspire des logiques d'assemblée du mouvement des indignés, reprenant certaines de ses pratiques, comme le fait de lever les mains et de les bouger pour exprimer son accord avec une prise de parole, il est toutefois globalement très différent, beaucoup plus rationnel et organisé, et s'apparente en fait de plus en plus à celui d'une organisation partisane classique. Les assemblées $d u$ « 15-M » étaient parfois interminables, sans véritable fil conducteur, laissant chacun intervenir quelquefois durant de longues minutes, en revenant parfois à des questions déjà traitées. À l'inverse, dans les réunions des cercles de Podemos, les militants annoncent désormais l'ordre du jour, disent dès le départ à quelle heure la réunion doit se terminer, répartissent et limitent les prises de parole, dans le but d'être le plus efficaces possible dans l'organisation du travail de mobilisation politique. D'autre part, dans la mesure où le parti s'institutionnalise rapidement en permettant à ses dirigeants locaux d'accéder à des mandats représentatifs, les conseils citoyens et leur direction sont devenus les principaux centres de pouvoir au détriment des cercles, qui réunissent certes les sympathisants mais n'influent pas réellement et de façon déterminante sur les décisions internes. C'est d'ailleurs pour cela que, passée l'effervescence initiale, beaucoup de cercles locaux sont désormais beaucoup moins, voire même plus du tout

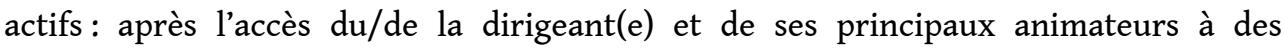
mandats locaux, ils s'investissent dans leurs nouvelles fonctions institutionnelles et le cercle ne vit plus réellement en dehors des périodes électorales. Ceux qui restent ont tendance à jouer le rôle d'un groupe de pression qui tente de contrôler l'activité de leurs élus locaux. À l'inverse, si aucun membre n'a accédé à un mandat représentatif, les réunions ont tendance à devenir de moins en moins régulières et on observe un fort désengagement militant depuis les municipales, lié à un cycle de désinvestissement.

\section{La sélection des candidats par des primaires ouvertes}

Podemos a aussi eu le mérite d'introduire en Espagne le débat sur la formation des listes électorales, en montrant, au moins initialement, que l'imposition par les partis de listes fermées n'est pas une fatalité et qu'il est possible au contraire de proposer aux citoyens des listes ouvertes qui permettent à de nouvelles individualités d'accéder à la représentation politique. Podemos soutient l'organisation de primaires ouvertes à l'ensemble de ses sympathisants pour élire les membres de son Conseil citoyen national et de ses conseils citoyens locaux dans chacune des 50 provinces espagnoles. Ses candidats lors des européennes de 2014 furent ainsi choisis par des primaires ouvertes à l'ensemble de ses sympathisants. N'importe quel citoyen, qui réussit à obtenir l'aval de l'un des cercles locaux de la formation, pouvait être candidat. Les candidats potentiels devaient simplement signer une déclaration sur l'honneur relative à l'absence d'antécédents pénaux pour corruption et une carte d'adhésion aux valeurs du 
parti. Cette procédure connut un large engouement populaire. Pour pouvoir présenter une liste aux européennes, les fondateurs devaient eux-mêmes recueillir 15000 signatures de citoyens, la procédure prévue par la législation espagnole pour les partis ne bénéficiant pas de représentation parlementaire. Le budget initial de Podemos fut de 25000 euros, mais atteignit rapidement 150000 euros après sa campagne de financement participatif. Chaque cercle local pouvait présenter jusqu'à trois candidats aux primaires : 150 personnes se présentèrent, puis le choix de la liste finale composée de 64 personnes ( 32 hommes et femmes) de même que leur ordre d'éligibilité sur la liste furent votés par l'ensemble des sympathisants lors d'un vote électronique qui mobilisa 20000 sympathisants. Même si ce processus fut ouvert et participatif, il bénéficia surtout à ceux dont la notoriété était la plus forte et qui surent le mieux mobiliser leurs réseaux militants tels que Pablo Iglesias et Teresa Rodríguez notamment.

En cherchant à favoriser la constitution de plateformes citoyennes lors des élections municipales de 2015, Podemos suscita aussi un fort engouement, notamment auprès des personnes les plus impliquées dans les collectifs locaux et les mouvements sociaux dans le contexte de la crise. Une multitude de plateformes telles que Ganemos Madrid, Aragon Si Se Puede ou Guanyem Barcelona dirigée par Ada Colau de la PAH, se sont ainsi formées au sein de nombreuses villes d'Espagne. Par exemple, dans la communauté autonome de Madrid, ce processus s'est largement diffusé : Ahora Getafe ; Leganemos ; Somos Alcala ; Ganemos Alcorcón ; Rivas Puede ; Mejoremos etc. Même si les ossatures de ces plateformes furent constituées par les militants locaux de Podemos, de simples sympathisants pouvaient aussi y participer. Mais le plus important ici est que les candidats et leur position sur chaque liste furent systématiquement choisis par des votes des groupes locaux. Les listes furent donc directement formées par les bases plutôt que par les dirigeants avant de rechercher l'assentiment des militants. Malgré une conjoncture défavorable au printemps 2015 marquée par l'affaire Monedero qui engendra un déclin important des intentions de vote du parti dans les sondages, la constitution participative des listes municipales lui permit de reprendre la main, d'obtenir $15 \%$ des voix en moyenne et des victoires symboliques majeures à Madrid et Barcelone notamment. La définition du programme électoral des européennes fut aussi assez démocratique et interactive : au programme de base élaboré par les fondateurs, chaque cercle pouvait incorporer des amendements qui étaient ensuite approuvés ou rejetés par les votes électroniques des sympathisants. On voit donc bien comment, derrière l'émergence électorale de Podemos, il y a aussi un processus participatif assez démocratique, ouvert et horizontal, tant dans la sélection des candidats que dans la définition du programme.

\section{Le Congrès constituant de Vistalegre et le renforcement de la centralisation du pouvoir}

Pour autant, les structures «nationales » du parti fonctionnent assez différemment et demeurent fortement centralisées autour du groupe dirigeant: de ce point de vue, Podemos apparait plus comme une organisation partisane « comme les autres ». Depuis sa fondation en 2014, il est ainsi possible d'observer un processus de renforcement de la verticale du pouvoir autour du cercle des fondateurs. Plusieurs épisodes ultérieurs aux élections européennes illustrèrent très bien les limites de sa démocratie interne. Ainsi, en juin 2014, lors de la nomination de "l'équipe technique", qui eut pour mission d'organiser la future assemblée constituante du parti, la démocratie interne fut une 
gageure : le groupe des fondateurs imposa d'en haut et sans consultation, le choix d'une élection entre des listes fermées de 25 personnes. Pablo Iglesias proposa la sienne, qui regroupait l'ensemble de ses proches, de telle sorte qu'il s'agissait formellement d'une " primaire », mais que la compétition fut inégale avec les autres listes potentielles, dont les candidats devaient faire face à un déficit de notoriété. Certains militants de base s'affrontèrent aux fondateurs et les cercles de Madrid demandèrent l'organisation de « listes ouvertes » comme lors des européennes ${ }^{49}$. L'annonce par lettre et en conférence de presse de l'organisation dès la semaine suivante de l'élection qui devait ratifier le choix de cette "équipe technique" fut très mal perçue. De nombreux militants critiquèrent le manque de temps pour constituer des listes alternatives. Le cercle de Lavapiés, un noyau fondateur qui devint rapidement assez critique, considéra en ce sens que « la proposition est précipitée et que nous n'avons pas assez d'information " Les représentants des assemblées de Vallecas, Moncloa, Arganzuela, Hortaleza, Usera, Chamartín, Fuencarral ou de la ville de Rivas (dont les assemblées étaient en pleine croissance), s'opposèrent fortement à la décision des fondateurs. Ceux des assemblées de La Latina, Moratalaz, ou encore du district de San Blas furent les plus virulents, en dénonçant "des pratiques internes oligarchiques" contredisant le discours d'exemplarité démocratique du parti. Certains journalistes évoquèrent un "énorme mal-être » et une "ambiance interne irrespirable ${ }^{51}$. À ces critiques, Juan Carlos Monedero répondit en défendant la nécessité de «donner une certaine autonomie à l'élite dirigeante " pour ne pas reproduire selon lui les erreurs du mouvement des indignés, « radicalement démocratique, mais radicalement inopérant $~^{52}$. Monedero alla même plus loin en alertant du risque de "coup d'État" au sein de l'organisation, accusant certains sympathisants de vouloir convertir le mouvement en « une gauche d'IU » et de le rendre " peu viable ». Au final, seul le cercle des infirmières présenta une liste alternative de 25 candidats composée notamment de syndicalistes et d'avocats et présentée comme " paritaire, pluri-territoriale et multidisciplinaire $»^{53}$. Le vote électronique pour départager les deux listes fermées, ouvert à l'ensemble des sympathisants de plus de 16 ans, eut lieu les 12 et 13 juin grâce au système "Agora Voting » et mobilisa 55000 votants. Sans surprise, la liste d'Iglesias obtint $86,8 \%$ des voix contre $10,34 \%$ pour la liste alternative regroupant le cercle des infirmières et une partie importante des cercles de Madrid.

30 Les structures nationales du parti furent de même définies par le groupe des fondateurs en juillet 2014, soumises à débat puis approuvées par les militants en novembre. Dans un Pré-document d'organisation, il fut proposé un parti doté d'un dirigeant et d'organes exécutifs centralisés autour d'un noyau dur de 15 personnes $^{54}$. Présentée comme " une organisation politique orientée à promouvoir et défendre la démocratie et les droits de l'homme ", les fondateurs proposèrent à leurs bases de faire de Podemos un parti combinant une structure nationale centralisée et des assemblées locales autogérées ${ }^{55}$. Il fut proposé que "l'Assemblée citoyenne", présentée comme l'organe principal de décision, se réunisse tous les trois ans lors d'un Congrès national : en son sein, tous les membres de Podemos, "ont le droit de participer avec la voix et le vote" pour « déterminer la ligne politique, le programme, approuver les statuts et les mécanismes de financement, de même que choisir leurs représentants $»^{56}$. Il fut aussi proposé de doter le parti d'un "Conseil citoyen» de 80 personnes, pensé comme un organe de contrôle permanent devant « donner une continuité à la ligne politique choisie dans les assemblées citoyennes et l'adapter aux circonstances du moment $»^{57}$. Enfin, le « Conseil de coordination ", composé de 15 personnes élues pour trois ans, constitue en fait la 
vraie direction du parti. Le référendum révocatoire fut aussi approuvé, à savoir que tant le secrétaire général, les membres du conseil citoyen national ou de coordination, que les dirigeants des groupes locaux peuvent être révoqués par un référendum convoqué par au moins $30 \%$ des affiliés du parti pour le niveau national, ou du conseil citoyen local concerné. Du point de vue de son organisation territoriale, l'unité de base du parti est le cercle, dont la fonction est de " promouvoir la participation, le débat et la relation active avec la société ». Dans les municipalités où il y a moins de 200 affiliés, "la structure n'aura pas d'autre organe que la propre assemblée citoyenne ", et dans celles de plus de 200, «l'organe de décision sera l'assemblée citoyenne intégrée par tous les membres des cercles $»^{58}$. Dans les communautés autonomes, le modèle municipal basé sur les cercles a été répliqué. Ces grands principes organisationnels, définis par l'élite dirigeante, consacrèrent la primauté d'un exécutif national autonome totalement libre de ses choix, et furent approuvés sans modification lors de l'Assemblée constituante de Vistalegre du 15 novembre 2014.

Dans ce processus d'institutionnalisation d'une nouvelle organisation partisane, la marginalisation des courants internes favorables au maintien d'un mouvement d'assemblée et critiques de la ligne des fondateurs illustre bien la "normalisation » progressive de Podemos. Représentant le courant interne "Sumando Podemos » avec les eurodéputées Teresa Rodríguez et Lola Sánchez, les militants Victor García, la féministe Beatriz Gimeno et le cercle des infirmières, l'eurodéputé Pablo Echenique retira sa candidature au secrétariat général après avoir mis en cause la méthode de vote, estimant qu'une compétition interne avec l'équipe de Pablo Iglesias était impossible ${ }^{59}$. Le courant «Construyendo Podemos» dirigé par Clara Marañón et Carolina Huelmo, qui réunit une dizaine de cercles très actifs (dont ceux de Madrid, Séville, Murcie, Ciudad Real, Alicante et Guipuscoa), émit de même des critiques. Le dilemme il est vrai, fut que les sympathisants durent soit voter en bloc pour la liste fermée proposée par les fondateurs, soit choisir un à un les 62 candidats au «Conseil citoyen ». Dans un tel système, le choix possible des candidats sur une base individuelle fut en compétition inégale avec la liste de Pablo Iglesias en raison du déficit de notoriété de la plupart des candidats. Le cercle des infirmières de Podemos fut d'ailleurs beaucoup plus critique, accusant ouvertement Iglesias de donner corps aux «premiers réflexes de caste» au sein du mouvement, montrant l'évolution rapide du parti vers une organisation dotée d'une direction autonome imposant des mécanismes de discipline et de hiérarchie partisane.

On voit donc bien comment l'essor de Podemos n'est pas juste le fruit du hasard, et se fonde aussi sur une volonté de rénovation des pratiques politiques à l'heure de l'internet et des réseaux sociaux, tant pour faire campagne que pour s'organiser en interne. Les nouvelles technologies sont activement utilisées comme des instruments de mobilisation et d'invitation au débat, ce qui permet de relayer plus effectivement les prises de position publiques des porte-paroles, et de mener de véritables « campagnes 2.0 ». L'usage des réseaux sociaux, la création d'assemblées autogérées, la définition participative des programmes, la sélection des candidats par des primaires sont autant d'évolutions qui visent à traduire en actes les principes d'une démocratie plus participative et horizontale. Mais cette article a surtout montré les tensions entre cette dimension citoyenne (participative) et la dimension organisationnelle (hiérarchique et 
formalisée), qui vise à ne pas reproduire les travers du mouvement des indignés, «radicalement démocratique, mais radicalement inopérant» pour reprendre l'expression de Juan Carlos Monedero.

D'une part, la trajectoire récente de Podemos a donc illustré un renforcement de la centralisation du pouvoir : après les municipales de mai 2015, la volonté d'imposer une discipline et une cohésion interne autour de la stratégie décidée par la direction a engendré un autoritarisme organisationnel croissant justifié sans cesse par l'impératif de conquête du pouvoir. Ainsi, une " primaire » a certes été organisée pour désigner les candidats aux législatives, mais lors d'un vote militant national, de telle sorte que les candidats ont ensuite été "parachutés » et répartis dans les circonscriptions par la direction, ce qui a entraîné des démissions locales en chaîne, notamment à Madrid et en Catalogne. En janvier 2017, il est possible de considérer que Pablo Iglesias dispose de proches qui adhérent à sa ligne politique à la tête de 12 des 17 conseils citoyens des communautés autonomes. Le Congrès Vistalegre II de février 2017 lui a permis de réaffirmer son leadership (ses sympathisants représentent désormais $60 \%$ des membres $\mathrm{du}$ Conseil citoyen), de maintenir une ligne d'opposition plus intransigeante et une vision plus "mouvementiste", où les élus du parti devraient selon lui être des « activistes institutionnels » faisant le lien entre la rue et les institutions, au détriment de la vision plus transversale prônée par Î́nigo Errejón, axée sur le travail institutionnel et la quête de crédibilité pour "faire moins peur» et incarner un parti de gouvernement pouvant diriger ou prendre part à une alternance future. Le secrétaire général a de même renforcé ses prérogatives en faisant approuver son pouvoir de destituer directement les dirigeants des conseils citoyens locaux ou de dissoudre ces derniers et de provoquer de nouvelles élections internes en cas de dissidence ouverte.

Mais, d'autre part, malgré ce renforcement de la centralisation du pouvoir, d'autres tendances montrent une volonté de faire évoluer certaines pratiques: en deux ans et demi depuis octobre 2014, Podemos a ainsi organisé 9 votes internes (auxquels il faut ajouter les votes des groupes locaux sur les enjeux les concernant, notamment dans la désignation des conseils citoyens), illustrant une fréquence très forte des consultations militantes sur les principales orientations du parti. Le nombre de sympathisants a aussi fortement progressé de 250000 en octobre 2014 à 413000 en janvier 2017, illustrant la volonté de créer un mouvement de masse, mais plus individualisé et au militantisme plus fluide. Même si les dirigeants estiment que les militants " actifs » se situent plutôt autour de 150000 personnes, ces chiffres demeurent assez impressionnants pour une organisation nouvelle : de fait, les votes sur le rejet de l'alliance avec Ciudadanos, puis sur le soutien à celle avec IU ont mobilisé environ 150000 militants à chaque fois. Même si leur contrôle pose question, les élus de Podemos continuent toujours à ne percevoir que trois fois le salaire minimum, le parti ayant institué une «Commission de garanties" en charge de veiller sur les pratiques internes. Ils signent aussi des déclarations d'intérêts, et ont parfois fait approuver une diminution des salaires de l'ensemble des élus dans les mairies qu'ils dirigent comme à Barcelone.

Pour conclure, il est donc possible de dire que Podemos n'est ni un parti politique classique, ni une forme d'organisation complétement nouvelle. Il cherche plutôt à concilier les deux logiques apparemment contradictoires du parti-mouvement et du parti traditionnel. À la base, il se structure comme un mouvement d'assemblées autonomes et autogérées, qui nomment leurs représentants, choisissent leurs priorités locales et leur stratégie d'alliance, ce qui explique leur géométrie variable en fonction 
des « écosystèmes » politiques locaux (alliance ou non avec le PSOE, IU etc.). L'absence de locaux "physiques" de nombreux cercles, les convocations et la diffusion des informations par les réseaux sociaux, ou encore le brouillage de la frontière entre sympathisants et militants par l'absence de cotisation, sont autant d'éléments qui distinguent pour l'instant Podemos des autres partis et qui rapprochent le fonctionnement de ses bases de celui d'un mouvement social se réunissant dans les espaces publics et axé sur l'organisation de manifestations de rue. Le "populisme" n'est pas une caractéristique intrinsèque du parti, dont les discours institutionnels s'apparentent plus à des discours d'opposition classiques, mais a été utilisé initialement comme une stratégie oppositionnelle et d'émergence, et demeure utilisé de temps à autre comme une stratégie de mobilisation.

Ses fondateurs sont d'ailleurs, on l'a vu, des entrepreneurs de cause professionnels de l'action militante de telle sorte que, même s'il a suscité l'engagement de profanes et d'une nouvelle génération de jeunes précaires en politique, Podemos se fonde d'abord sur l'expertise sociologique et stratégique de politologues qui mettent leurs connaissances au service d'une entreprise de refondation d'une gauche alternative. Même s'il s'appuie sur des procédures visant à rendre son fonctionnement interne plus démocratique, il serait toutefois trompeur de le qualifier de "parti d'assemblée ». Certes, à travers la fédération d'assemblées locales, la consultation par vote des militants sur les grandes orientations du parti et la revendication de transparence en publiant ses comptes sur internet, Podemos cherche à mettre en application les principes de la démocratie participative et directe. La systématisation de l'utilisation des moyens de communication liés aux réseaux sociaux lui permet de plus de communiquer et de faire campagne à des coûts très faibles. Mais la ligne politique adoptée lors du Congrès fondateur a intronisé un secrétaire général doté de larges pouvoirs sur les assemblées locales, et a confié quasiment l'ensemble du pouvoir interne à un groupe dirigeant où l'on retrouve la plupart des fondateurs. Le parti est certes doté d'un "conseil citoyen" assez élargi, mais aussi d'un exécutif dirigeant, d'une hiérarchie et d'une discipline comme les partis traditionnels. Fédération d'assemblées locales autogérées dont les décisions sont soumises à l'approbation des bases, Podemos est donc toutefois aussi un parti au leadership très marqué. Il se structure en fait différemment suivant le niveau considéré : l'autonomie très forte du groupe dirigeant sur les grandes orientations nationales, sur lesquelles les militants de base ont en fait très peu de pouvoir, contraste avec le fort degré d'autogestion des assemblées locales. En alliant centralisation du pouvoir et autogestion il cherche en fait à combiner une stratégie nationale avec une autonomie locale autour de représentants dont la vocation désirée est celle de se convertir en « activistes institutionnels ».

\section{NOTES}

1. . Entretien avec Beatriz, infirmière, meeting de Móstoles, 11 mai 2015.

2. . Thomas Fromentin et Stéphanie Wojcik (dir.), Le profane en politique. Compétences et engagements du citoyen, Paris, L'Harmattan, 2008. 
3. . Pablo Iglesias, « Understanding Podemos », New Left Review, $n^{\circ}$ 93, mai 2015, p. 20.

4. . Yann Raison $d u$ Cleuziou, «Le secours catholique et les forums sociaux : une politisation incrémentale (2003-2010) », Critique Internationale, vol. 1, n 5, 2011, p. 73-89.

5. . « Lola Sánchez, candidata a las primarias de Podemos », DiarioSur, 5 mars 2014.

6. . « Los otros diputados de Podemos », Público, 28 mai 2014.

7. . Tania González, eurodéputée de Podemos, fiche biographique du Parlement européen, décembre 2016.

8. . «El político más influyente del que nunca has oído hablar », El Mundo, 24 septembre 2015.

9. . Antonio Negri, Le Pouvoir constituant. Essai sur les alternatives de la modernité, Paris, PUF, 1997.

10. . Luis Giménez, «En la Facultad, radicales y mayoritarios », in Ana Domínguez et Luis Giménez (dir.), Claro que Podemos. De La Tuerka a la esperanza del cambio en España, Madrid, Los Libros del Lince, 2014, p. 19.

11. Acción de Contrapoder en la Facultad de Políticas de la Complutense, You Tube, 2006.

12. . « Espacio Alternativo becomes Izquierda Anticapitalista and decides to stand in European elections ", International Viewpoint, 24 novembre 2008.

13. . "Quién es quién en Izquierda Anticapitalista, el partido que mueve los hilos dentro de Podemos », Vozpópuli, 16 juin 2014.

14. . Pierre-André Taguieff, «Le populisme et la science politique. Du mirage conceptuel aux vrais problèmes ", Vingtième Siècle, n 56, 1997, p. 8.

15. . Ernesto Laclau, La raison populiste, Paris, Le Seuil, 2008.

16. . Fabio García Lupato, « Les usages et l'intériorisation de l'Europe au sein de la compétition parlementaire en Espagne et en Italie », in Mathieu Petithomme (dir.), L'européanisation de la compétition politique nationale, Grenoble, PUG, 2011, p. 76-101.

17. . Ernesto Laclau, Op. Cit., 2008, p. 96.

18. . Giovanni Sartori, Partis et systèmes de partis: un cadre d'analyse, Bruxelles, Éditions de l'université de Bruxelles, [1967] 2011.

19. . Otto Kircheimer, "The waning of opposition in parliamentary regimes ", Social Research, vol. $24, \mathrm{n}^{\circ} 1,1957$, p. $128-129$.

20. . Enquête CIS, janvier 2016.

21. . Enquête CIS, juin 2014, et Ibid.

22. . Alexandre Dorna, Le populisme, Paris, Presses universitaires de France, 1999, p. 5.

23. . Pablo Iglesias, « Podemos : el partido de las clases populares », El País, 30 novembre 2015.

24. . Patrick Charaudeau, «Réflexions pour l'analyse du discours populiste », Mots. Les langages du politique, n 97, 2011, p. 101-116.

25. . « Podemos quiere politizar el dolor », El País, 11 octobre 2016.

26. . Pablo Iglesias, cité dans « ¿Hacia dónde camina Podemos? », El Mundo, 15 septembre 2014, p. 15.

27. . Max Weber, Le savant et le politique, Paris, Plon, [1919] 2002.

28. . Otto Kircheimer, "The transformation of the West European party systems ", in Joseph La Palombara et Myron Weiner (dir.), Political Parties and Political Development, Princeton, Princeton University Press, 1966, p. 177-200.

29. . « El espejo griego de Syriza », El País, 23 novembre 2014.

30. Sur une échelle classique de positionnement, 0 correspond à l'extrême-gauche, 5 au centre et 10 à l'extrême-droite. Cf. sondage El País/Metroscopia, 2 novembre 2014.

31. . Sylvain Crépon, Alexandre Dézé et Nonna Mayer, Les faux-semblants du Front National. Sociologie d'un parti politique, Paris, Presses de Sciences-Po, 2015.

32. . «Un proyecto económico para la gente », programme économique, élections européennes, avril 2014.

33. . Propuesta de Programa Económico, elecciones legislatives, 2015, p. 4. 
34. . Pablo Iglesias (dir.), Una nueva transición. Materiales del año del cambio, Madrid, Akal, Pensamiento Crítico, 2015, p. 162.

35. . « Podemos propone nuevos impuestos, gravar la banca y bajar el IVA », El País, 31 octobre 2015.

36. . « Podemos expone sus propuestas económicas al empresariado », El País, 6 novembre 2015.

37. . « Las europeas empiezan por la red », El País, 19 mars 2014.

38. . El Mundo, 15 juin 2014, p. 3.

39. . « La comunicación digital de Podemos », El Mundo, 5 juin 2014.

40. . Luis Gómez, « Las redes de arrastre de Podemos », El País, 30 mai 2014.

41. . Rita Maestre, citée dans El País, 30 mai 2014.

42. . Ibid.

43. . «La infraestructura de Podemos vive en internet », El País, 15 novembre 2014.

44. . Entretien avec Fernando, Lavapiés, 25 février 2015.

45. . Entretien avec Àngel, siège « numérique » de Podemos, Plaza de España, 24 février 2015.

46. . Héloïse Nez, Podemos. De l'indignation aux élections, Paris, Éditions les Petits Matins, 2015, p. $35-40$.

47. . « Pablo Echenique : 'Hay que reactivar los círculos », El Mundo, 14 octobre 2016.

48. . Entretien avec Lidia, commerçante, 38 ans, Ciutat Vella, Valence, 12 septembre 2015.

49. . « Las bases de Podemos se enfrentan a sus fundadores para exigir democracia interna », El País, 9 juin 2014.

50. . « Militantes de Podemos se rebelan contra la dirección del partido », El Mundo, 8 juin 2014.

51. . El Mundo et La Vanguardia, 8 juin 2014.

52. . Juan Carlos Monedero, cité dans El País, 9 juin 2014.

53. . " El círculo de enfermeras de Podemos presenta una lista alternativa », El País, 12 juin 2014.

54. . Borrador de ponencia de organización, Podemos, juillet 2014.

55. . « Podemos se organizará como un partido con líder y órganos ejecutivos », El Mundo, 25 juillet 2014.

56. . Borrador de ponencia de organización, Op. Cit., 2014, p. 2

57. Ibid., p. 2.

58. . Ibid., p. 4

59. . « Las renuncias de Echenique », El Periódico de Catalunya, 24 octobre 2014, p. 5

INDEX

Mots-clés : Podemos, mouvements sociaux, partis politiques, gauche alternative

\section{AUTEUR}

\section{MATHIEU PETITHOMME}

Maître de conférences en science politique à l'université de Bourgogne/Franche-Comté, spécialiste de l'Espagne contemporaine. 